Missed Diagnosis

\title{
Pulmonary embolism: a cause of acute confusion in the elderly
}

\author{
Jonathan E. Shaw and Peter W. Belfield \\ Department of Medicine for the Elderly, Chapel Allerton Hospital, Leeds LS7 4RB, UK
}

\begin{abstract}
Summary: Large numbers of elderly patients are admitted to hospital with an acute confusional state. In many of these, the underlying cause is easily found, but in a proportion, correct diagnosis is very difficult. Two cases of acute confusion due to hypoxia are presented, in which the diagnosis of pulmonary embolic disease was made late. The importance of considering this in all patients presenting with acute confusion and the value of blood gas analysis is discussed.
\end{abstract}

\section{Introduction}

Acute confusion is very common in the elderly and cerebral hypoxia due to pneumonia or cardiac failure is a well recognized cause for this. ${ }^{1}$ The other common cause of hypoxia, pulmonary embolism, is clinically difficult to diagnose and all too often missed or only found at post mortem. ${ }^{2}$ We report two elderly patients with multiple pulmonary emboli, who presented with acute confusion and neuropsychiatric problems.

\section{Case reports}

Case 1

A 77 year old, previously fit and independent woman, was admitted to hospital with a 6-day history of confusion. Her medication included piroxicam, atenolol and chlorthalidone. There was no history of trauma. Examination was unremarkable and her Hodkinson ${ }^{3}$ abbreviated mental test score (AMTS) was 2/10. Initial investigations showed a plasma sodium of $122 \mathrm{mmol} / 1$ and a urea of $28.6 \mathrm{mmol} / 1$. Despite correction of these and stopping the diuretic, there was no improvement in her mental state. Other investigations for treatable causes of confusion (including full blood count, plasma viscosity, liver function tests, syphilis serology, serum B12, folate, thyroid function tests, blood cultures, urine culture, chest X-ray and electrocardiogram) were all normal. There were no focal neurological signs and she remained very

Correspondence: P.W. Belfield, M.R.C.P. Accepted: 12 January 1991 mobile with a tendency to wander. Ten days after admission, she had 5 very brief convulsions. Three of these were focal. An urgent brain CT scan showed involutional changes, with no intracranial mass, haematoma or infarct. Electroencephalo gram showed no signs of a mass or epileptie features.

During the next week she remained confused (AMTS 3/10), did not stay in bed and tried to leave the ward on several occasions. She then became breathless and hypotensive and had florid signs of right heart failure. Chest X-ray showed a band shadow at the right lung base and the electrocardiogram showed a sinus tachycardia and right heart strain. Arterial blood gas analysis revealed a $\mathrm{PO}_{2}$ of $6.8 \mathrm{kPa}$ and a $\mathrm{PCO}_{2}$ of $4.0 \mathrm{kPa}$. The clinical diagnosis of pulmonary embolus was confirmed by an isotope lung scan, which showed multiple unmatched perfusion defects. She was anticoagulated and over the next few weeks her mental state steadily improved. At outpatient follow up at 6 months, she remains fully orientated (AMTS 9/10) and lives independently at home.

\section{Case 2}

An 85 year old woman with a history of polymyalgia rheumatica had three separate but stereotyped admissions over the course of one year with acute confusion. The confusion was associated with hallucinations, such as being attacked and chopped into little pieces and being thrown into the sewers. During each of these admissions her polymyalgia was quiescent.

On the first two occasions, she was treated for a presumed chest infection. These episodes were 
diagnosed as pneumonia on the findings of breathlessness, cough and coarse crepitations at the bases. Chest X-ray on each of these occasions showed right basal shadowing which, on serial films, did not change and was thought to be due to pleural thickening. No sputum was available for culture. Blood gases on the first occasion showed mild hypoxia with a $\mathrm{PO}_{2}$ of $7.7 \mathrm{kPa}$ and $\mathrm{PCO}_{2}$ of $5 \mathrm{kPa}$. Both times her confusion resolved (AMT 10/10) by the time of discharge.

On the third occasion, she was admitted with breathlessness but as before, had no chest pain or haemoptysis. Chest $\mathrm{X}$-ray again showed right sided pleural shadowing and the electrocardiogram was in sinus rhythm with a normal axis. Blood gases showed hypoxia $\mathrm{PO}_{2}$ of $6.8 \mathrm{kPa}$ with normocarbia, $\mathrm{PCO}_{2} 4.8 \mathrm{kPa}$. Review of previous episodes raised the possibility of pulmonary embolic disease. This was confirmed by a ventilation perfusion scan which showed large areas of mis-match and she was anticoagulated.

Her mental state improved and during the following 2 years, there have been no further episodes and she has remained independent at home (AMTS 10/10). It seems likely that her acute confusional episodes, often associated with hallucinations, were the result of pulmonary emboli rather than simply due to chest infections.

\section{Discussion}

We believe that our cases represent acute confusional states secondary to episodes of hypoxia, resulting from repeated pulmonary emboli. In both cases the diagnosis was made late. Following anticoagulation, it seems likely that there were no more emboli and the patients' cognitive function returned to normal.

The clinical diagnosis of pulmonary embolus is often difficult and only $30 \%$ of fatal pulmonary emboli are correctly diagnosed in life. ${ }^{2}$ In the elderly, where there is often concomitant cardiorespiratory disease, the problem is especially difficult and only $10 \%$ of the over 70 s had the correct diagnosis made before death. ${ }^{2}$ Problems with the diagnosis of pulmonary embolus occur due to the wide variety of clinical presentations. ${ }^{4-11}$ A number of studies from over 25 years ago ${ }^{4-6}$ showed that up to $5 \%$ of pulmonary emboli may present with neurological manifestation such as fits or hemiplegia. Despite this evidence that patients with signs and symptoms of cerebral hypoxia alone may have a pulmonary embolus, it continues to be missed. Indeed, more recent reviews ${ }^{7-11}$ do not mention confusion as a presentation.

Acute confusion in the elderly is a common presentation of a very wide variety of illnesses. The underlying cause is often uncovered by clinical assessment and simple investigations. However, if the diagnosis remains unclear, it is all too easy to attach unproven labels such as stroke or psychiatric illness. A significant proportion of these patients may have pulmonary emboli, and as in these cases, correct diagnosis and treatment may improve mental function and increase their chances of full recovery.

It is important to consider pulmonary embolus in all cases of acute confusion, even if, as in the first case, there are no signs or symptoms referable to the respiratory system. Blood gas analysis is a simple investigation and is almost always abnormal, especially in this group of patients who are confused because of hypoxia. We therefore recommend that blood gas analysis be an early investigation in all confusional states of uncertain cause.

\section{References}

1. Weatherall, D.J., Ledingham, J.G.G. \& Warrell, D.A. Oxford Textbook of Medicine. Oxford University Press, Oxford, 1987.

2. Goldhaber, S.Z., Hennekens, C.H., Evans, D.A., Newton, E.C. \& Godleski, J.J. Factors associated with correct antemortem diagnosis of major pulmonary embolism. Am J Med 1982, 73: 822-825.

3. Hodkinson, H.M. Hodkinson test. Evaluation of a mental score for the assessment of mental impairment in the elderly. Age Ageing, 1972, 1: 233-238.

4. Sagall, E.L., Bornstein, J. \& Wolff, L. Clinical syndrome in patients with pulmonary embolism. Arch Intern Med 1945 , 76: $234-238$.

5. Israel, H.L. \& Goldstein, F. The varied clinical manifestations of pulmonary embolism. Ann Intern Med 1957, 47: $202-226$

6. Whittington Gorham, L. A study of pulmonary embolism. Arch Intern Med 1961, 108: 76-90.

7. Bell, W.R., Simon, T.L. \& DeMets, D.L. The clinical features of submassive and massive pulmonary emboli. Am J Med 1977, 62: 355-360.

8. National Co-op Study. The Urokinase Pulmonary Embolism Trial. Circulation 1973, 47 (Suppl II): 1-108.

9. Sasahara, A.A., Cannilla, J.E., Morse, R.L., Sidd, J.J. \& Tremblay, G.M. Clinical and physiologic studies in pulmonary thromboembolism. Am J Cardiol 1967, 20: 10-20.

10. Sutton, G.C., Honey, M. \& Gibson, R.V. Clinical diagnosis of acute massive pulmonary embolism. Lancet 1969, i: $271-273$.

11. Barritt, D.W. \& Jordan, S.C. Clinical features of pulmonary embolism. Lancet 1961, i: 729-732. 